\title{
Changes in microstructural parameters of NB4D2 silk fibres due to electron irradiation: $\mathrm{X}$-ray line profile analysis
}

\author{
SANGAPPA*, S ASHA, P PARAMESWARA ${ }^{\dagger}$ and R SOMASHEKAR ${ }^{\dagger}$ \\ Department of Studies in Physics, Mangalore University, Mangalagangotri 574 199, India \\ ${ }^{\dagger}$ Department of Studies in Physics, University of Mysore, Manasagangotri, Mysore 570 006, India
}

MS received 21 May 2010

\begin{abstract}
The present study is concerned with changes of microcrystalline parameters in NB4D2 (Bombyx mori) silk fibres, due to electron irradiation. The irradiation process was performed in air at room temperature using $8 \mathrm{MeV}$ electron beam at different dose rates: $0,25,50$ and $75 \mathrm{kGy}$, respectively. $\mathrm{X}$-ray recording of these irradiated samples and the line profile analysis were carried out. The crystal imperfection parameters such as crystallite size $\langle N\rangle$, lattice strain $(g$ in $\%)$ and surface weighted crystallite size $\left(D_{s}\right)$ were computed and compared with other physical parameters in order to asertain the changes that have crept into these irradiated fibres. Exponential, lognormal and Reinhold functions for the column length distributions have been used for the determination of these parameters.
\end{abstract}

Keywords. Irradiation; microstructural parameters; WAXS, fibre.

\section{Introduction}

Silk is a fascinating fibre which has applications in biotechnological and biomedical fields because of its intrinsic property having been made up of proteins. In addition, there is an age old importance of silk in the manufacture of textiles (Miller et al 1999). There is a continued interest in improving the properties of silk and various methods have been employed for this purpose. One such method is electron irradiaton. In this context, it is quite important to know microstructural changes in silk fibres due to electron irradiation. These parameters determine the property and strength of the fibres. Very little studies have been carried out on these lines except for the chemical effects on these fibres (Tsukuda et al 1994, 1995, 1996; Mohanthy et al 1995; Freddi et al 1996; Kavahara et al 1996; Somashekarappa et al 1996). Okuyama et al (1988) reported crystal structure for silk-I and silk-II fibres. Somashekarappa et al (1998) reported the effect of degumming and dye processing on the microstructural parameters in pure Mysore silk, Nistari, NB7 and NB18 silk fibres. Sangappa et al (2004, 2005b, 2010) reported microstructural parameters in Hosa Mysore (HM), Pure Mysore Silk (PMS), Nistari, C-nichi and changes of microstructural parameters in electron-irradiated NB7 silk fibres. Takeshita et al (2000) studied the effect of electron beam irradiation on silk fibres. Effects of gamma irradiation on biodegradation of Bombyx mori silk fibres have been carried out by Kojthung's (2008) group.

\footnotetext{
*Author for correspondence (syhalabhavi@yahoo.co.in)
}

When a polymer is subjected to irradiation by ionizing radiation such as gamma rays, X-rays or accelerated electrons, various effects like modification and degradation are expected from the interaction of beam with polymer. At the microscopic level, the polymer degradation is characterized by macromolecular chain splitting, creation of low mass fragments, production of free radicals, oxidation and cross-linking. These affect the macroscopic properties like mechanical strength, colour, electrical conductivity and so on (Wang et al 1987). The resulting change in the properties of the polymer may be favourable in certain applications of silk. In radiation chemistry, polymers are classified into two types: scission polymers and cross-linking polymers, and most biopolymers are classified as scission polymers (Charlesky 1995). In the present study, we have irradiated NB4D2 Bombyx mori silk fibre samples with $8 \mathrm{MeV}$ electron beam. Experiments have been carried out for various dose rates. Line profile analysis (LPA) of X-ray Bragg's reflections from such irradiated samples has been carried out. An attempt has been made to bring out the structure-property relation in these irradiated NB4D2 silk fibres.

\section{Experimental}

\subsection{Sample preparation}

For our study we have used raw NB4D2 silk fibre belonging to Bombyx mori family which comes under the classification of bivoltine on the basis of shape, colour, denier and life cycle of the fibres/cocoons. Cocoons were 
collected from the germplasm stock of the Department of Sericulture, University of Mysore, India, which were then cooked in boiling water $\left(100^{\circ} \mathrm{C}\right)$ for 2 min to soften the sericin and transferred to water bath at $65^{\circ} \mathrm{C}$ for $2 \mathrm{~min}$. Then the cocoons were reeled in warm water with the help of mono cocoon reeling equipment EPPROUVITE. The characteristic features of these fibres are that they are white in colour with an average filament length of $1100 \mathrm{~m}$ and thickness of the silk fibre was $2 \cdot 1$ denier. These fibres were mounted on rectangular frame in just taut condition which does not involve any mechanical stretching of fibres. The whole process, starting from reeling to mounting of fibres, does not involve any type of mechanical deformation.

\subsection{Electron irradiation}

Irradiation of samples was carried out at Microtron Centre; Mangalore University, using the electron beam (by lanthanum hexa fluorite source). The monochromatic beam was made to fall on samples kept at a particular distance with the beam features which are listed in table 1 .

The dose delivered to different samples was measured by keeping alanine dosimeter with sample during irradiation.

\section{$2.3 X$ X-ray diffraction measurements}

XRD diffractograms of the fibre samples were recorded using a Rigaku Miniflex-II X-ray diffractometer with $\mathrm{Ni}$ filtered, $\operatorname{CuK} \alpha$ radiation of wavelength, $\lambda=1.5406 \AA$, with a graphite monochromator. The scattered beam was focused on a detector. The samples were scanned in the $2 \theta$ range $10-50^{\circ}$ with a step size of $0.01^{\circ}$ and scans are given in figure 1.

\section{Theory}

Microstructural parameters such as crystal size $(\langle N\rangle)$ and lattice strain ( $g$ in \%) are usually determined by employing Fourier method of Warren and Averbach (1950), and Warren (1969). The intensity of a profile in the direction joining the origin to the centre of the reflection can be expanded in terms of Fourier cosine series

Table 1. Specifications of the electron beam accelerator and irradiation conditions.

\begin{tabular}{lll}
\hline 1. & Beam energy & $8 \mathrm{MeV}$ \\
2. & Beam current & $20 \mathrm{~mA}$ \\
3. & Pulse repetition rate & $50 \mathrm{~Hz}$ \\
4. & Pulse width & $2 \cdot 2 \mu \mathrm{s}$ \\
5. & Distance source to sample & $30 \mathrm{~cm}$ \\
6. & Dose range & $0-75 \mathrm{kGy}$ \\
7. & Atmosphere & $\mathrm{Air}$ \\
8. & Temperature & $24^{\circ} \mathrm{C}$ \\
\hline
\end{tabular}

$$
I(s)=\sum_{n=-\infty}^{\infty} A(n) \cos \left\{2 \pi n d\left(s-s_{0}\right)\right\},
$$

where the coefficients of the harmonics, $A(n)$, are functions of size of the crystallite and disorder of the lattice. Here, $s$ is $\sin (\theta) /(\lambda), s_{0}$ being the value of $s$ at the peak of a profile, $n$ the harmonic order of coefficient and $d$ the lattice spacing. The Fourier coefficients can be expressed as

$$
A(n)=A_{\mathrm{s}}(n) \cdot A_{\mathrm{d}}(n) .
$$

For a paracrystalline material, $A_{\mathrm{d}}(n)$ can be obtained, with Gaussian strain distribution (Hall and Somashekar 1991),

$$
A_{\mathrm{d}}(n)=\exp \left(-2 \pi^{2} m^{2} n g^{2}\right) .
$$

Here, $m$ is the order of reflection and $g=(\Delta d / d)$ the lattice strain. Normally one also defines mean square strain $\left\langle\varepsilon^{2}\right\rangle$, which is given by $g^{2} / n$. This mean square strain is dependent on $n$, whereas not $g$ (Popa et al 1995; Ribarik et al 2001). For a probability distribution of column lengths, $P(i)$, we have

$$
A_{\mathrm{s}}(n)=1-\frac{n d}{D}-\frac{d}{D}\left[\int_{0}^{n} i P(n) \mathrm{d} i-n \int_{0}^{n} P(i) \mathrm{d} i\right]
$$

where $D=\langle N\rangle d_{h k l}$ is the crystallite size and $i$ the number of unit cells in a column. In the presence of two orders of reflections from the same set of Bragg planes, Warren and Averbach (1950) have shown a method of obtaining the crystal size $(\langle N\rangle)$ and lattice strain $(g$ in $\%)$. But in polymer it is very rare to find multiple reflections. So, to determine the finer details of microstructure, we approximate the size profile by simple analytical function for $P(i)$ by considering only the asymmetric functions. Another advantage of this method is that the distribution function differs along different directions. Whereas, a single size distribution function that is used for the whole pattern fitting, which we feel, may be inadequate to describe polymer diffraction patterns (Popa and Balzar et al 1995; Ribarik et al 2001; Scardi and Leoni 2001). Here it is emphasized that the Fourier method of profile analysis (single order method used here) is quite a reliable one as per the recent survey and results of Round Robin test conducted by IUCr (Balzar 2002). In fact, for refinement, we have also considered the effect of background by introducing a parameter [see for details, regarding the effect of background on the microcrystalline parameters (Somashekar et al 1989)]. For the sake of completeness, we reproduce the following equations which are used in the computation of microstructural parameters.

\subsection{Exponential distribution}

It is assumed that there are no columns containing fewer than $p$ unit cells and those with more decay 

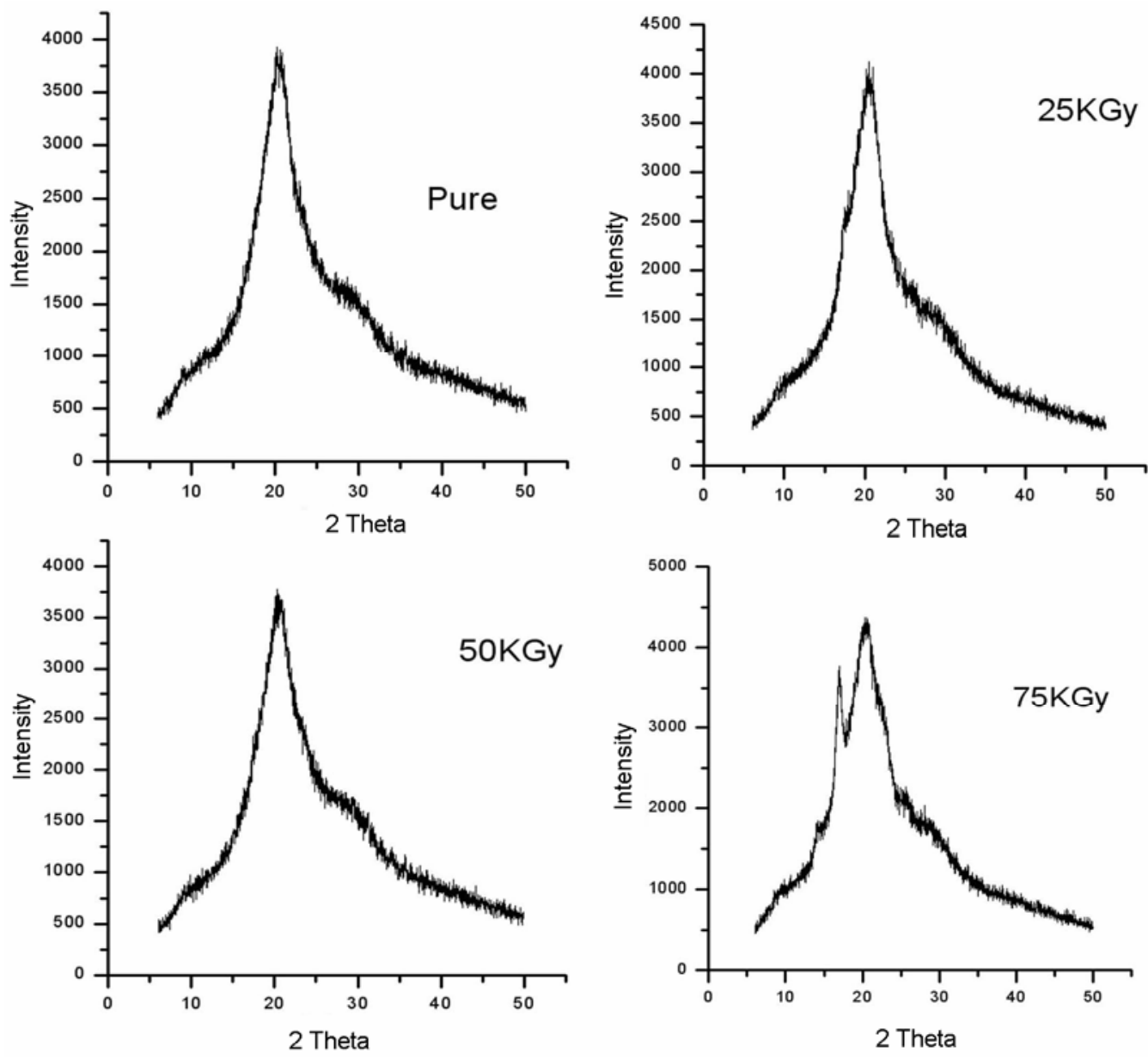

Figure 1. XRD scans of pure and $8 \mathrm{MeV}$ electron-irradiated polymer samples.

exponentially. Thus, we have (Somashekar and Somashekarappa 1997)

$$
P(i)=\left\{\begin{array}{ll}
0 ; & \text { if } p<i \\
\alpha \exp \{-\alpha(i-p)\} ; & \text { if } n \geq i
\end{array},\right.
$$

where $\alpha=1 /(N-p)$. Substituting this in (4), we get

$$
A_{\mathrm{s}}(n) \begin{cases}A(0)(1-n /\langle N\rangle) ; & \text { if } n \leq p \\ A(0)\{\exp [-\alpha(n-p)]\} /(\alpha N) ; & \text { if } n \geq p\end{cases}
$$

Here, $\alpha$ is width of the distribution function, $i$ the number of unit cells in a column, $n$ the harmonic number, $p$ the smallest number of unit cells in a column and $\langle N\rangle$, the number of unit cells counted in a direction perpendicular to the $(h k l)$ Bragg plane.

\subsection{Lognormal distribution}

The lognormal distribution function is given by

$$
P(i)=\frac{1}{(2 \pi)^{1 / 2} \sigma} \frac{1}{i} \exp \left\{-\frac{[\log (i / m)]^{2}}{2 \sigma^{2}}\right\},
$$

where $\sigma$ is the variance and $m$ the median of the distribution function.

Substituting for $P(i)$ in (4) and simplifying (5), we get

$$
\begin{aligned}
A_{\mathrm{s}}(n)= & \frac{m^{3} \exp \left[(9 / 4)\left(2^{1 / 2} \sigma\right)^{2}\right]}{3} \\
& \times \operatorname{erfc}\left[\frac{\log (|n| / m)}{2^{1 / 2} \sigma}-\frac{3}{2} 2^{1 / 2} \sigma\right] \\
& -\frac{m^{2} \exp \left(2^{1 / 2} \sigma\right)^{2}}{2}|n| \operatorname{erfc}\left[\frac{\log |n| / m}{2^{1 / 2} \sigma}-2^{1 / 2} \sigma\right] \\
& +\frac{|n|^{3}}{6} \operatorname{erfc}\left[\frac{\log (|n| / m)}{2^{1 / 2} \sigma}\right] .
\end{aligned}
$$

The above equation is the one used by Ribarik et al (2001). The maximal value, $A_{\mathrm{s}}(0)$, is given by

$$
A_{\mathrm{s}}(0)=\frac{2 m^{3} \exp \left[(9 / 4)\left(2^{1 / 2} \sigma\right)^{2}\right]}{3} \text {. }
$$


The area-weighted number of unit cells in a column is given by

$$
\langle N\rangle_{\text {surf }}=\frac{2 m \exp \left[(5 / 4)\left(2^{1 / 2} \sigma\right)^{2}\right]}{3},
$$

and the volume-weighted number of unit cell in a column is given by

$$
\langle N\rangle_{\mathrm{vol}}=\frac{3 m \exp \left[(7 / 4)\left(2^{1 / 2} \sigma\right)^{2}\right]}{4} .
$$

\subsection{Reinhold distribution}

With the exponential distribution function, $P(i)$ rises discontinuously at $p$, from zero to its maximum value. In contrast, the Reinhold function allows a continuous change by putting

$$
P(i)=\left\{\begin{array}{ll}
0 ; & \text { if } i \leq p \\
\beta^{2}(i-p) \exp \{-\beta(i-p)\} & \text { if }>p
\end{array},\right.
$$

where $\beta=2 /(N-p)$ and substituting theses in (4), we obtain

$$
A_{\mathrm{s}}(n)= \begin{cases}A(0)(1-n /\langle N\rangle) ; & \text { if } n \leq p \\ {[A(0)(n-p+2 / \beta) / N]\{\exp [-\beta(n-p)]\} ;} & \text { if } n \geq p\end{cases}
$$

where $\beta$ is width of the distribution which has been varied to fit the experimental results. $p$ is the smallest number of unit cells in a column, $\langle N\rangle$ the number of unit cells counted in a direction perpendicular to the $(h k l)$ Bragg plane, $d$ the spacing of the $(h k l)$ planes; $\lambda$ the wavelength of X-rays used, $i$ the number of unit cells in a column; $n$ the harmonic number and $D_{\mathrm{s}}$ the surface weighted crystal size $\left(\langle N\rangle d_{h k l}\right)$.

All the distribution functions were put to test in order to find out the most suitable crystal size distribution function for the profile analysis of the X-ray diffraction. The procedure adopted for the computation of the parameters is as follows. Initial values of $g$ and $N$ were obtained using the method of Nandi et al (1984). With the Nandi et al (1984) method, we have estimated the parameters, which are initial values. These are only rough estimates, so the refinement procedure must be sufficiently robust to start with such values. Here we compute

$$
\Delta^{2}=\left[I_{\mathrm{cal}}-\left(I_{\mathrm{exp}}+B G\right)\right]^{2} / n p t,
$$

where $B G$ represents the error in the background estimation (figure 2), $n p t$ the number of data points in a profile, $I_{\text {cal }}$ the intensity calculated using (1)-(13) and $I_{\exp }$ the experimental intensity. The values of $\Delta$ were divided by half the maximum value of intensity so that it is expressed relative to the mean value of intensities, and then minimized.

\section{$3.4 \quad X$-ray profile analysis}

For the analysis, we have used X-ray diffraction data in the above equations to simulate the intensity profile by varying the necessary parameters till one gets a good fit with the experimental profile. For this purpose, a multidimensional algorithm SIMPLEX is used for minimization (Press et al 1986). We have used pure and $8 \mathrm{MeV}$ electron beam irradiated NB4D2 silk fibre samples. The computed crystal imperfection parameters along with reported physical parameters are given in table 2 for different distribution functions for each of the samples.

\section{Results and discussion}

Figures 3(a-d), 4(a-d) and 5(a-d) show the simulated and experimental profiles for $8 \mathrm{MeV}$ electron irradiated and pure polymer samples for Bragg's clear reflections (110) using different asymmetric functions. The simulated profile was obtained with the above equations using appropriate model parameters. This procedure was followed for all the other samples treated at different radiation doses for polymer samples. The computed microcrystalline parameters such as crystallite size $\langle N\rangle$ (number of unit cells), lattice strain $g$ in $\%$, width of the crystallite size distribution $(\alpha)$ and standard deviation are given in table 2 . It is evident from table 2 that all the asymmetric distributions used, give more or less similar results. By and

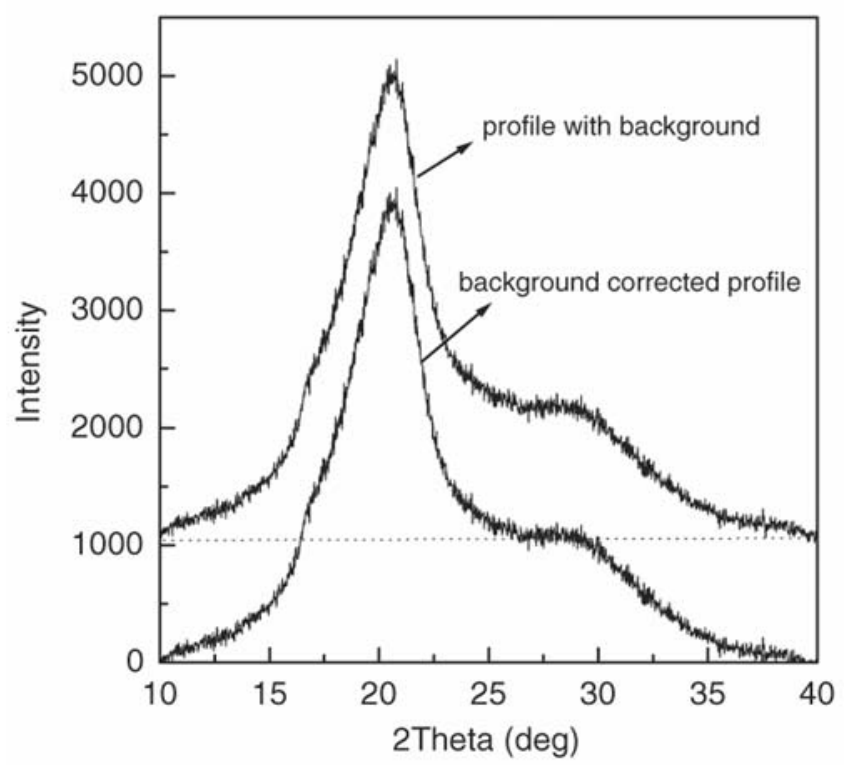

Figure 2. Profiles with background and background corrected NB4D2 silk fibre. 
Table 2. Microstructural parameters of electron-irradiated polymer samples computed by various distribution functions.

\begin{tabular}{|c|c|c|c|c|c|c|c|c|c|c|c|c|c|c|}
\hline \multirow[b]{2}{*}{ Sample } & \multirow[b]{2}{*}{$2 \theta$} & \multirow[b]{2}{*}{$d_{h k l}$} & \multicolumn{4}{|c|}{ Exponential } & \multicolumn{4}{|c|}{ Reinhold } & \multicolumn{4}{|c|}{ Lognormal } \\
\hline & & & $\langle N\rangle$ & $g$ in $\%$ & $D_{\mathrm{s}}(\AA)$ & Delta & $\langle N\rangle$ & $g$ in $\%$ & $D_{\mathrm{s}}(\AA)$ & Delta & $\langle N\rangle$ & $g$ in \% & $D_{\mathrm{s}}(\AA)$ & Delta \\
\hline $0 \mathrm{kGy}$ & $20 \cdot 62$ & $4 \cdot 256$ & $\begin{array}{l}9.56 \pm \\
0.86\end{array}$ & $\begin{array}{l}1 \cdot 0 \pm \\
0 \cdot 10\end{array}$ & $40 \cdot 69$ & 0.09 & $\begin{array}{l}9 \cdot 80 \pm \\
0 \cdot 88\end{array}$ & $\begin{array}{l}2 \cdot 0 \pm \\
0 \cdot 18\end{array}$ & $41 \cdot 71$ & 0.09 & $\begin{array}{l}9.90 \pm \\
0.78\end{array}$ & $\begin{array}{l}3 \cdot 0 \pm \\
0.54\end{array}$ & $42 \cdot 13$ & $0 \cdot 18$ \\
\hline 25 kGy & $20 \cdot 56$ & $4 \cdot 345$ & $\begin{array}{l}6.45 \pm \\
0.58\end{array}$ & $\begin{array}{l}2 \cdot 0 \pm \\
0 \cdot 18\end{array}$ & 28.03 & 0.09 & $\begin{array}{l}6 \cdot 56 \pm \\
0 \cdot 66\end{array}$ & $\begin{array}{l}4 \cdot 0 \pm \\
0 \cdot 40\end{array}$ & $28 \cdot 50$ & $0 \cdot 10$ & $\begin{array}{l}6 \cdot 60 \pm \\
0.79\end{array}$ & $\begin{array}{l}3 \cdot 0 \pm \\
0 \cdot 36\end{array}$ & $28 \cdot 68$ & $0 \cdot 12$ \\
\hline 50 kGy & $20 \cdot 28$ & 4.256 & $\begin{array}{l}6 \cdot 38 \pm \\
0.45\end{array}$ & $\begin{array}{l}2 \cdot 0 \pm \\
0 \cdot 14\end{array}$ & $27 \cdot 15$ & $0 \cdot 07$ & $\begin{array}{l}6 \cdot 34 \pm \\
0 \cdot 51\end{array}$ & $\begin{array}{l}3 \cdot 0 \pm \\
0 \cdot 24\end{array}$ & $26 \cdot 98$ & $0 \cdot 08$ & $\begin{array}{l}6 \cdot 30 \pm \\
0.69\end{array}$ & $\begin{array}{l}4 \cdot 0 \pm \\
0 \cdot 44\end{array}$ & $26 \cdot 81$ & $0 \cdot 11$ \\
\hline 75 kGy & $20 \cdot 66$ & $4 \cdot 326$ & $\begin{array}{l}6 \cdot 19 \pm \\
0 \cdot 49\end{array}$ & $\begin{array}{l}3 \cdot 0 \pm \\
0 \cdot 24\end{array}$ & $26 \cdot 78$ & $0 \cdot 08$ & $\begin{array}{l}6 \cdot 20 \pm \\
0.55\end{array}$ & $\begin{array}{l}4 \cdot 0 \pm \\
0 \cdot 36\end{array}$ & $26 \cdot 82$ & 0.09 & $\begin{array}{l}6 \cdot 20 \pm \\
0 \cdot 68\end{array}$ & $\begin{array}{l}4 \cdot 0 \pm \\
0 \cdot 44\end{array}$ & $26 \cdot 82$ & $0 \cdot 11$ \\
\hline
\end{tabular}
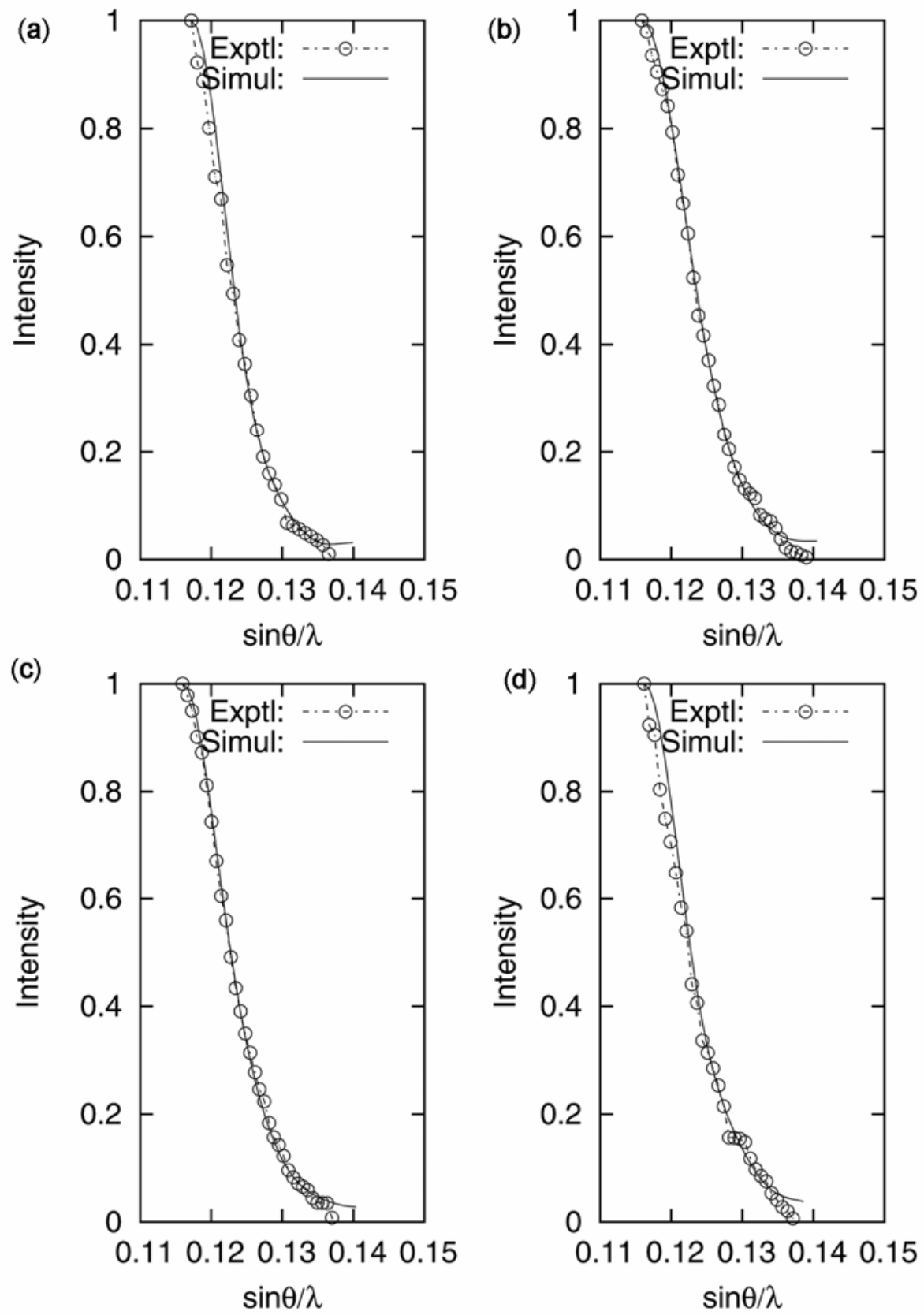

Figure 3. (a-d) Experimental and simulated intensity profiles of X-ray reflection of silk fibre samples obtained with exponential column length distribution function. 

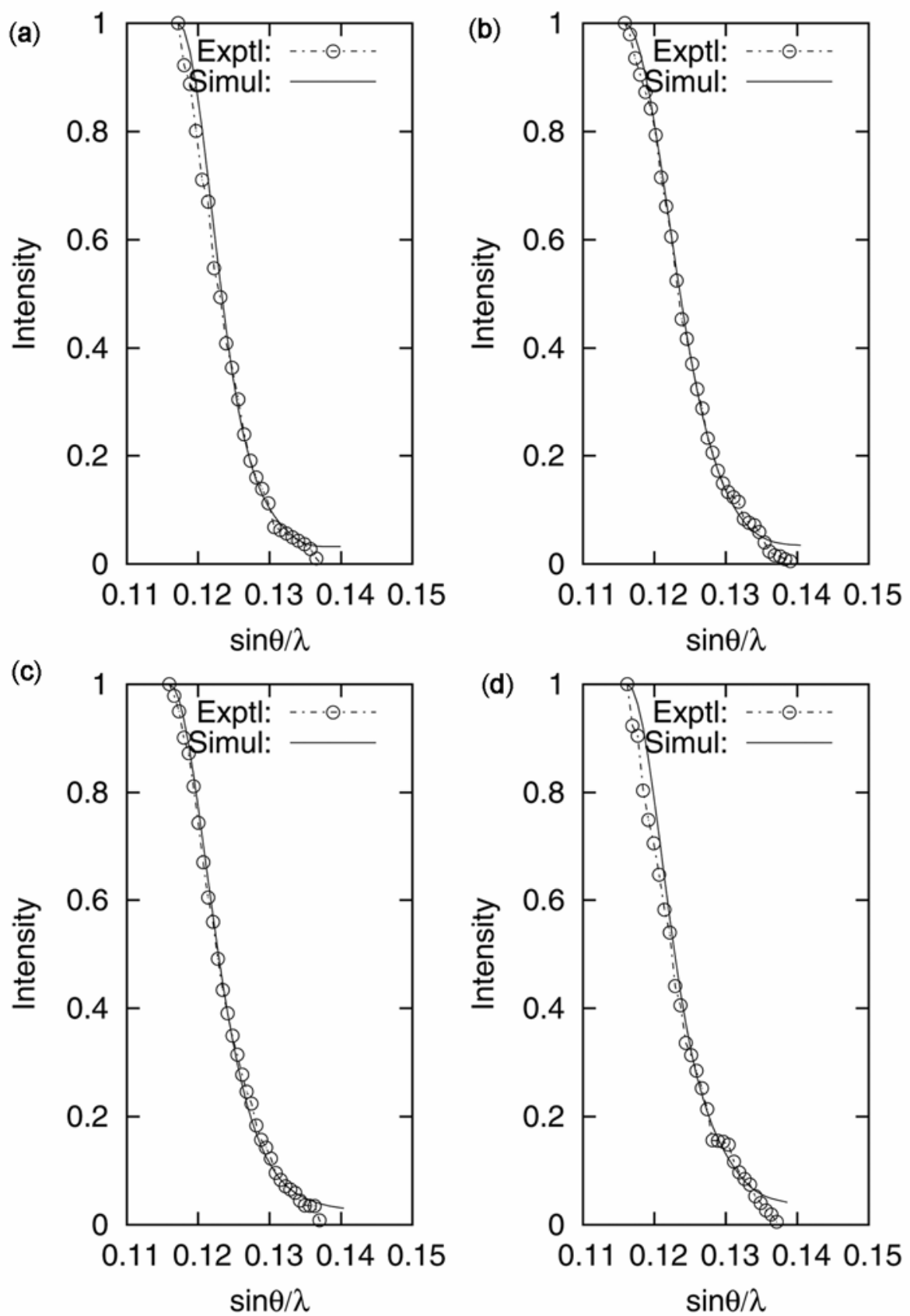

Figure 4. (a-d) Experimental and simulated intensity profiles of X-ray reflection of silk fibre samples obtained with Reinhold column length distribution function.

large, exponential distribution function gives a better fit than Reinhold/lognormal distributions. Here we would like to emphasize that the standard deviation in all the cases for the microstructural parameters are given in table 2 as delta. Since exponential distribution function gives a better fit than others, we used the corresponding results given in table 2 to infer some important conclusions. They are: (i) value of the surface weighted crystallite size, $D_{\text {s }}(\AA)$, decreases as irradiation dose increases and (ii) value of the crystallite size $\langle N\rangle$ is more for unirradiated polymers.
Irradiation of polymers mainly causes two important changes: (I) Degradation of the polymer, wherein main chain scission takes place, leading to low molecular weight polymer and (II) cross-linking of small polymer units leading to the formation of a rigid three-dimensional network, wherein a high molecular weight polymer is produced. Both these effects cause changes in physical properties. Degradation of polymer leads to loss in mechanical strength, whereas cross-linking improves the physical properties. It was found that the influence of electron beam irradiation with an increase in radiation 

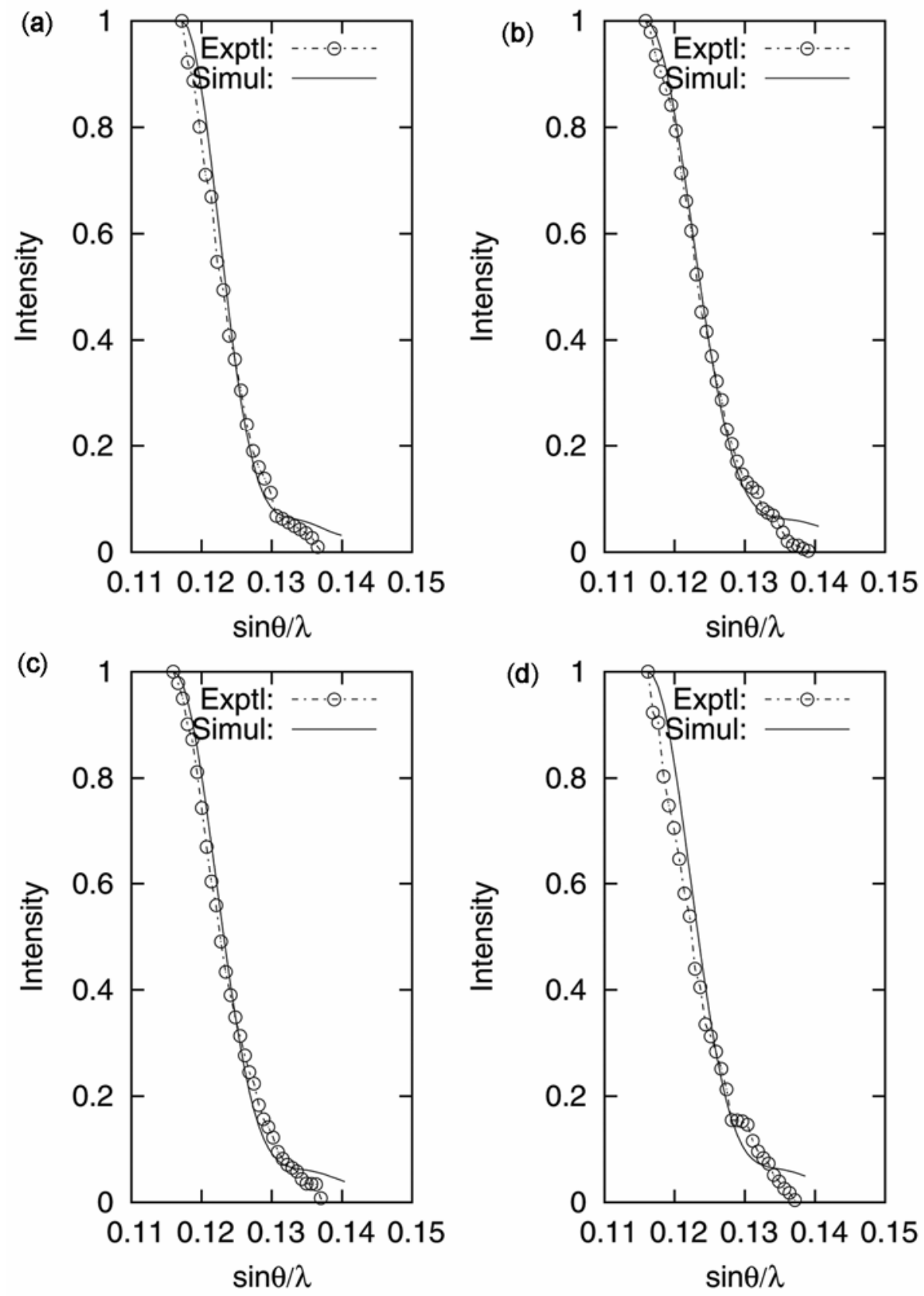

Figure 5. (a-d) Experimental and simulated intensity profiles of X-ray reflection of silk fibre samples obtained with lognormal column length distribution function.

dose and an increase in degree of cross-linking results in increase in modulus and $T_{\mathrm{g}}$ (Banik and Bhowmick 1999). In the case of many other polymeric materials, ionizing radiation may cross-link them, cause chain scission or affect their surface. Quite often these effects may occur simultaneously. The final result depends on the nature of the material, on the dosage, dosage rate and energy of radiation. From table 2 it is evident that the crystallite size decreases as irradiation dose increases. Normally the strength of the fibres, irrespective of natural or man- made, increases with increase in crystallite size (Lee et al 1995). This suggests that the unirradiated fibre will have more strength than irradiated fibres. Our results quantify the changes in terms of microstructural parameters using line profile analysis.

The variation of lattice strain $(g)$ lies between 1.0 and $4.0 \%$ in the case of exponential distribution for polymer samples. From table 2, it is clear that the lattice strain and its variation for various values of the radiation doses $(\mathrm{kGy})$ in polymer samples are very small. 


\section{Conclusions}

From the wide angle X-ray scattering (WAXS) study of electron irradiated NB4D2 silk fibre (Bombyx mori) samples, we have observed that even though there is not much change in the position of the X-ray reflections, a significant change in the values of microstructural parameters occurs. The significant change in microstructural parameters in polymer is due to the effect of electron irradiation. This causes the degradation of small regions of polymer network units leading to the formation of low ordered regions of polymer network. We have shown that among the three asymmetric crystallite size distributions, exponential gives a better fit in polymer samples. The only justification for the good fit that we observed with exponential distribution in these polymers can be interpreted on the basis of extensive usage of this function in condensed matter to explain various phenomena such as dielectric relaxation, luminescence decay law and other physical properties.

\section{Acknowledgements}

The authors are thankful to the University Grants Commission, New Delhi, for providing financial assistance through a project F. No. 33-14/2007 (SR).

\section{References}

Balzar D 2002 IUCr News Lett. 22814

Banik Indranil and Bhowmick Anil K 1999 Radiat. Phys. Chem. 54135

Charlesky A 1995 J. Polym. Sci.15 263

Freddi G, Massafra M R, Beretta S, Shibata S, Gotch Y, Yasui H and Sukuda M T 1996 J. Appl. Polym. Sci. 6011

Hall I H and Somashekar R 1991 J. Appl. Cryst. 124105

Kavahara Y, Shioya M and Takaku A 1996 J. Appl. Polym. Sci. 5951

Kojthung A, Meesilpa P, Sudatis B, Treeratanapiboon L, Udomsangpetch R and Oonkhanond B 2008 Int. Biodeterior. Biodegrad. 62487
Lee K G, Barton Jr R and Schultz J M 1995 J. Polym. Sci. B33 1

Miller Lance D, Putthanarat S and Adoms WW 1999 Biomacromolecules $\mathbf{2 4} 159$

Mohanthy N, Das H K, Mohanthy P and Mohanthy E 1995 J. Macromol. Sci. A32 1103

Nandi R K, Kho H K, Schlosberg W, Wissler G, Cohen J B and Crist Jr B 1984 J. Appl. Cryst. 1722

Okuyama K, Takanashi K, Nakajima Y, Hasegawa Y, Hirabayashi K and Nishi N 1988 J. Seric. Sci. 5723

Popa N C and Balzar D 1995 J. Appl. Crystallogr. 35338

Press W, Flannery B P, Teukolsky S and Vetterling W T (eds) 1986 Numerical recipes (Cambridge: Cambridge University Press)

Ribarik R, Ungar T and Gubicza J 2001 J. Appl. Cryst. 34669

Sangappa, Okuyama K and Somashekar R 2004 J. Appl. Polym. Sci. 913045

Sangappa, Mahesh S S and Somashekar R 2005a J. Biosci. 30 259

Sangappa, Mahesh S S, Somashekar R and Subramanya G 2005b J. Polym. Res. 12465

Sangappa, Asha S, Ganesh Sanjeev, Subramanya G, Parameswara P and Somashekar R 2010 J. Appl. Polym. Sci. 115 2183

Scardi P and Leoni M 2001 Acta Crystallogr. A57 604

Somashekar R and Somashekarappa H 1997 J. Appl. Crystal$\log r .130147$

Somashekar R, Hall I H and Carr P D 1989 J. Appl. Cryst. 22363

Somashekarappa H, Selvakumar N, Subramaniam V and Somashekar R 1996 J. Appl. Polym. Sci. 591677

Somashekarappa H, Nadgir G S, Somashekar T H, Prabhu J and Somashekar R 1998 Polymer 39209

Takeshita H, Ishida K, Kamiishi Y, Yoshii F and Kume T 2000 Macromol. Mater. Eng. 283126

Tsukuda M, Gotch Y, Nagura M, Minoura N, Kasai N and Freddi G 1994 J. Polym. Sci. B32 961

Tsukuda M, Freddi G and Monti P 1995 J. Appl. Polym. Sci. 33 1995

Tsukuda M, Obo M, Kato H, Freddi G and Zenetti F 1996 J. Appl. Polym. Sci. 601619

Wang G, Pao G, Dow L, Jiang S and Dai Q 1987 Nucl. Instrum. Meth. B27 410

Warren B E 1969 X-ray diffraction (New York: AddisionWesley)

Warren B E and Averbach L 1950 J. Appl. Phys. 21595 